Boise State University

ScholarWorks

\title{
$5-2013$
}

\section{Noncontacting Benchtop Measurements of the Elastic Properties of Shales}

Thomas E. Blum

Boise State University

Ludmila Adam

Boise State University

Kasper van Wijk

Boise State University 


\title{
Noncontacting benchtop measurements of the elastic properties of shales
}

\author{
Thomas E. Blum¹, Ludmila Adam², and Kasper van Wijk
}

\begin{abstract}
We evaluated a laser-based noncontacting method to measure the elastic anisotropy of horizontal shale cores. Whereas conventional transducer data contained an ambiguity between phase and group velocity measurements, small laser source and receiver footprints on typical core samples ensured group velocity information in our laboratory measurements. With a single dense acquisition of group velocity versus group angle on a horizontal core, we estimated the elastic constants $c_{11}, c_{33}$, and $c_{55}$ directly from ultrasonic waveforms, and $c_{13}$ from a least-squares fit of modeled to measured group velocities. The observed significant $\mathrm{P}$-wave velocity and attenuation anisotropy in these dry shales were almost surely exaggerated by delamination of clay platelets and microfracturing, but provided an illustration of the new laboratory measurement technique. Although challenges lay ahead to measure preserved shales at in situ conditions in the lab, we evaluated the fundamental advantages of the proposed method over conventional transducer measurements.
\end{abstract}

\section{INTRODUCTION}

Shale formations comprise about $75 \%$ of the clastic fill of sedimentary basins, and recent interest to exploit shales as potential reservoirs requires better understanding of their elastic behavior. Accurate estimation of elastic moduli has implications in understanding response and distribution of stress in shales (Dewhurst and Siggins, 2006; Holt et al., 2011), as well as in hydraulic fracturing (Suarez-Rivera et al., 2006). Shales can be represented as thin isotropic layers with a symmetry axis, also called transversely iso- tropic (TI), or hexagonal. Wave propagation in a transversely isotropic medium can be described with five elastic constants, and the ratios among these parameters quantify the rock anisotropy (Thomsen, 1986; Tsvankin, 2001).

Shale anisotropy in the laboratory has been widely studied with transducer ultrasonic systems at variable saturation and pressure conditions (Jones and Wang, 1981; Vernik and Nur, 1992; Johnston and Christensen, 1995; Hornby, 1998; Wang, 2002; Dewhurst and Siggins, 2006; Bayuk et al., 2009; Holt et al., 2011; Sondergeld and Rai, 2011). Three directions of wave propagation on core samples are the minimum requirement to estimate the five elastic constants of the stiffness tensor. For measurements with transducers, this is achieved by cutting three samples at $0^{\circ}, 45^{\circ}$, and $90^{\circ}$ from the shale layers (Vernik and Nur, 1992; Hornby, 1998; Sondergeld and Rai, 2011) or by using one core plug with transducers attached at these three angles (Wang, 2002; Dewhurst and Siggins, 2006). Frequency dependence of the elastic constants has recently been the topic of stress-strain and ultrasonic laboratory experiments. Results are mixed, probably due to sample heterogeneity and saturation conditions. Duranti et al. (2005) conclude that the measured West Africa shales display frequency dispersion, whereas Sarker and Batzle (2010) observe no changes in the elastic stiffness constants with frequency in an organic rich shale. The measurements described above have been mostly performed on saturated shales, whereas the measurements presented in this paper are currently only recorded on dry samples. Recently, Miller et al. (2012) show that for fast anisotropic formations, sonic logs measure the group velocity and that measurements at different angles from a deviated well can be used to directly estimate all the elastic constants.

Here, we propose a new methodology to measure the directional dependence of elastic velocity and amplitude on one horizontal shale core plug by acquiring dense and high-quality velocity data. The method uses a noncontacting laser source and receiver (Pouet and Rasolofosaon, 1990; Scales and Malcolm, 2003; Blum et al.,

Manuscript received by the Editor 7 August 2012; revised manuscript received 23 October 2012; published online 29 April 2013.

${ }^{1}$ Boise State University, Department of Geosciences, Physical Acoustics Laboratory, Boise, Idaho, USA. E-mail: thomasblum@u.boisestate.edu.

${ }^{2}$ Boise State University, Department of Geosciences, Physical Acoustics Laboratory, Boise, Idaho, USA and The University of Auckland, Institute of Earth Science and Engineering (IESE) and the School of Environment, Auckland, New Zealand. E-mail: mila.adam@boisestate.edu.

${ }^{3}$ Boise State University, Department of Geosciences, Physical Acoustics Laboratory, Boise, Idaho, USA and The University of Auckland, Department of Physics, Auckland, New Zealand. E-mail: kaspervanwijk@boisestate.edu.

(c) 2013 Society of Exploration Geophysicists. All rights reserved. 
2010; Bretaudeau et al., 2011; Blum, 2013), which has the following advantages over transducer acquisition: (1) transducer coupling and ringing do not affect the data, (2) sampling is as dense as one trace every $0.25 \mathrm{~mm}$, (3) acquisition is fast and automated, (4) for the case of TI media, the fast and slow velocity directions do not need to be known or assumed before acquisition, and (5) the group velocity is measured, regardless of sample size. The current system measures the sample properties at room conditions; however, our laboratory is developing capabilities to measure rock samples under pressure.

Guilbaud and Audoin (1999) and Ogi et al. (2003) use laser ultrasonics to measure the elastic properties of anisotropic materials. Scales and Malcolm (2003) observe directional P-wave velocity anisotropy in a fractured granite sample by using laser sources and receivers. Velocity (Dewangan et al., 2006) as well as amplitude (Zhu et al., 2007) or polarization (Lebedev et al., 2011) measurements in phenolic materials are studied using transducer sources and a laser receiver. In this manuscript, we outline the procedure and summarize observations of source-receiver laser ultrasonic measurements on horizontally cored shales, and we compare these data to transducer ultrasonic measurements.

Figure 1 is a photograph of the two horizontal shale samples measured in this study. Sample MSH is an oil shale acquired from an outcrop in Montana with a density of $1.70 \mathrm{~g} / \mathrm{cm}^{3}$, and shale SHC has a density of $2.40 \mathrm{~g} / \mathrm{cm}^{3}$. Because the samples are measured dry and at room conditions, the anisotropy estimates do not represent that of shales in situ. However, the purpose here is to describe a new methodology to estimate elastic constants, which can be implemented on preserved samples and under reservoir conditions, in future work. Because we are focused on the methodology, we do not analyze the petrographical characteristics of these dry samples. A follow-up study on preserved samples will be integrated with detailed sample analysis.

\section{THEORETICAL DESCRIPTION}

A medium is called vertical transversely isotropic (VTI) if the symmetry axis is defined in the vertical direction for a specific coordinate system ( $x_{3}$ in our study). The stiffness tensor for a VTI media has five independent elastic constants, namely, $c_{11}, c_{13}, c_{33}, c_{55}$ and $c_{66}$ (Tsvankin, 2001). The velocities of a P- $\left(V_{\mathrm{P} 0}\right)$ and two polarized S- $\left(V_{\mathrm{SV} 0}\right.$ and $\left.V_{\mathrm{SH} 0}\right)$ waves propagating along the symmetry axis $\left(x_{3}, \theta=0^{\circ}\right.$; see Figure 2$)$ are defined as

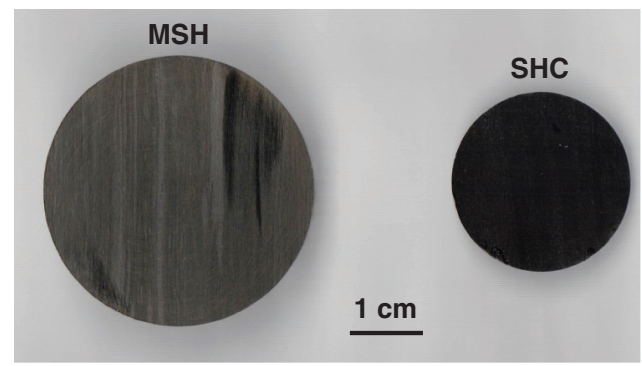

Figure 1. Top view of the two shale samples measured in this study. Lamination is clearly observed in sample MSH, whereas sample SHC has visible cracks, but these are not captured in this photograph.

$$
V_{\mathrm{P} 0}=\sqrt{\frac{c_{33}}{\rho}}, \quad V_{\mathrm{SV} 0}=\sqrt{\frac{c_{55}}{\rho}}, \quad V_{\mathrm{SH} 0}=\sqrt{\frac{c_{55}}{\rho}},
$$

where $\rho$ is the rock's bulk density. For propagation parallel to the symmetry plane $\left(\theta=90^{\circ}\right)$, the velocities correspond to

$$
V_{\mathrm{P} 90}=\sqrt{\frac{c_{11}}{\rho}}, \quad V_{\mathrm{SV} 90}=\sqrt{\frac{c_{55}}{\rho}}, \quad V_{\mathrm{SH} 90}=\sqrt{\frac{c_{66}}{\rho}} .
$$

The P-wave anisotropy of a VTI medium can be described with Thomsen's parameters $\epsilon$ and $\delta$ (Thomsen, 1986). The parameter $\epsilon$ quantifies the velocity difference for wave propagation along and perpendicular to the symmetry axis, whereas $\delta$ controls the $\mathrm{P}$-wave propagation for angles near the symmetry axis

$$
\begin{gathered}
\epsilon=\frac{c_{11}-c_{33}}{2 c_{33}}, \\
\delta=\frac{2\left(c_{13}+c_{55}\right)^{2}-\left(c_{33}-c_{55}\right)\left(c_{11}+c_{33}-2 c_{55}\right)}{2 c_{33}^{2}} .
\end{gathered}
$$

These expressions are general, and $\delta$ has not been simplified for the weak anisotropy case $(\delta \ll 1)$. The P-wave phase velocity as a function of phase angle $(\theta)$ and the elastic constants for a VTI medium is (Tsvankin, 2001)

$$
V_{\mathrm{P}}(\theta)=\sqrt{\frac{\left(c_{11}+c_{55}\right) \sin ^{2} \theta+\left(c_{33}+c_{55}\right) \cos ^{2} \theta+\mathrm{D}}{2 \rho}},
$$

where

$$
\begin{aligned}
\mathrm{D}= & \left\{\left[\left(c_{11}-c_{55}\right) \sin ^{2} \theta-\left(c_{33}-c_{55}\right) \cos ^{2} \theta\right]^{2}\right. \\
& \left.+4\left(c_{13}+c_{55}\right)^{2} \sin ^{2} \theta \cos ^{2} \theta\right\}^{1 / 2}
\end{aligned}
$$

and the constant $c_{13}$ is physically bound by the following relation (Tsvankin, 2001):

$$
c_{13, \max }=\sqrt{c_{33} c_{11}} .
$$

Although most transducer experiments measure the phase velocity (Dellinger and Vernik, 1994; Hornby, 1998), our experimental setup, described in the following section, measures the propagation along a straight line between the source and receiver. This results in a measurement of the group velocity as a function of group angle. Tsvankin (2001) shows that the $\mathrm{P}$-wave group velocity $U_{P}$ is given as a function of phase angle $\theta$ by

$$
U_{P}(\theta)=V_{\mathrm{P}}(\theta) \sqrt{1+\left(\frac{1}{V_{\mathrm{P}}(\theta)} \frac{d V_{\mathrm{P}}}{d \theta}\right)^{2}},
$$

and the group angle $\psi$ is given by

$$
\tan \psi=\tan \theta\left(1+\frac{\frac{1}{V_{\mathrm{P}}(\theta)} \frac{d V_{\mathrm{P}}}{d \theta}}{\sin \theta \cos \theta\left(1-\frac{\tan \theta}{V_{\mathrm{P}}(\theta)} \frac{d V_{\mathrm{P}}}{d \theta}\right)}\right) .
$$

Equations 8 and 9 highlight that for $\theta=0^{\circ}$ and $\theta=90^{\circ}$ (the "slow" and "fast" directions, respectively), we have $\psi=\theta$ and $U_{P}=V_{\mathrm{P}}$. 
Thus, of the five independent elastic constants, $c_{13}$ is the only parameter sensitive to whether we measure phase or group velocity. We estimate $c_{13}$ from equations 5 and 6 , but because we measure group angles and velocities, we use equations 8 and 9 to compare the measured group velocity data to predicted phase velocity.

\section{LABORATORY SETUP}

In the laboratory, we use laser-based ultrasonics to study wave propagation in various settings. We use a high-energy pulsed $\mathrm{Nd}$ :YAG laser (1064 $\mathrm{nm}$ wavelength, $10 \mathrm{~ns}$ pulses, $0.4 \mathrm{~J} /$ pulse) to excite ultrasonic waves via thermoelastic expansion (Scruby and Drain, 1990). When an energy pulse from the laser hits an optically absorbing surface, part of that energy is absorbed and converted into heat. This results in localized thermal expansion, which in turn generates elastic waves in the ultrasonic range. Here, we partially focus the laser source beam on the shale samples, getting a circular source with a diameter of approximately $6 \mathrm{~mm}$.

We measure the resulting waves with a laser ultrasonic receiver. Our adaptive laser interferometer is based on a continuous-wave doubled neodymium-doped yttrium aluminum garnet (Nd:YAG) laser, generating a $250-\mathrm{mW}$ beam at a wavelength of $532 \mathrm{~nm}$. The receiver uses two-wave mixing in a photorefractive crystal to determine the displacement of the sample surface. This receiver measures the out-of-plane (vertical) component of the wavefield, as well as one in-plane (horizontal) component, with a spot size at the sample surface in the order of $50 \mu \mathrm{m}$. It is calibrated to output the absolute displacement field in nanometers (see Blum et al. [2010] for a complete description of the laser receiver). The frequency response is flat between $20 \mathrm{kHz}$ and $20 \mathrm{MHz}$, and it accurately detects displacements of the order of Ångströms. Because our shale samples are dark materials, reflective tape on the sample enhances the amount of light reflected back to the laser receiver. We use $90-\mu \mathrm{m}$ thick aluminum tape. At the range of frequencies considered here, the tape transmits bulk waves with a flat frequency response, but it introduces a time delay of $\sim 0.02 \mu$ s for the out-of-plane channel, and of $\sim 0.16 \mu$ s for the in-plane channel, both accounted for during data processing (Blum, 2013).

As sketched in Figure 2, the shale plug is mounted in the center of a rotational stage and the source and receiver beams are aligned on antipodes for a transmission experiment. The lasers and the sample are positioned on an optical bench with vibration isolation, and a photograph of the experimental setup is presented in Figure 3. The rotational stage is computer controlled, and the output of the interferometer is acquired at 10 megasamples/s and digitized at 16-bit precision. By using a PCI digital oscilloscope card, we are able to fully automate the data acquisition. To increase the signal-to-noise ratio, 200 measurements are summed for each angular position. The acquisition time is approximately $4 \mathrm{~h}$ per sample. This experimental setup provides a direct measurement of the propagation along the ray direction, and hence it leads to an estimation of the group velocity as a function of the group angle, as shown in Figure 1.1 of Tsvankin (2001).

\section{RESULTS}

Wavefields as a function of group angle are presented in Figures 4 and 5 for samples MSH and SHC, respectively. The amplitudes represent absolute particle motion, and data are filtered between $50 \mathrm{kHz}$ and $5 \mathrm{MHz}$. The dominant frequency of the measured waves is $500 \mathrm{kHz}$ in the fast direction and approximately $250 \mathrm{kHz}$ in the

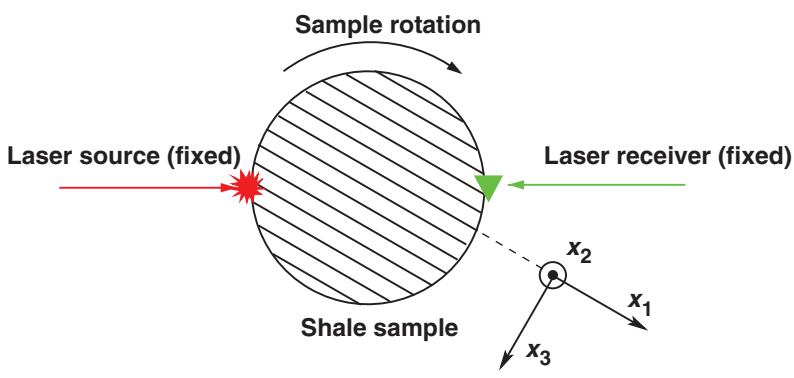

Figure 2. Top-view schematic of the experimental setup with respect to the defined axis directions, where $x_{3}$ is the axis of rotational symmetry and the plane $\left(x_{1}, x_{2}\right)$ is parallel to the beddings. The phase and group angles $(\theta$ and $\psi$, respectively, as used in equations 8 and 9) are zero when the source and receiver are aligned with $x_{3}$.

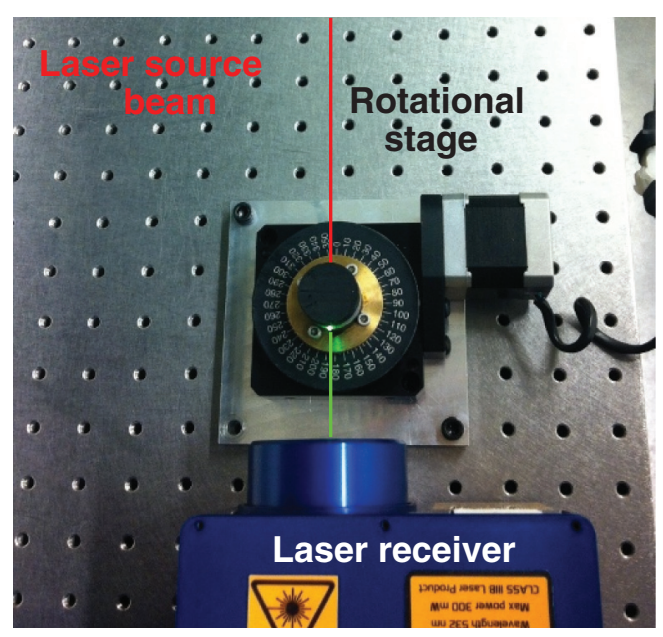

Figure 3. Photograph of the experimental setup from the top. The source laser beam propagates from the upper left corner of the photo and reflects off a mirror toward the sample, whereas the laser receiver is at the bottom of the photo.

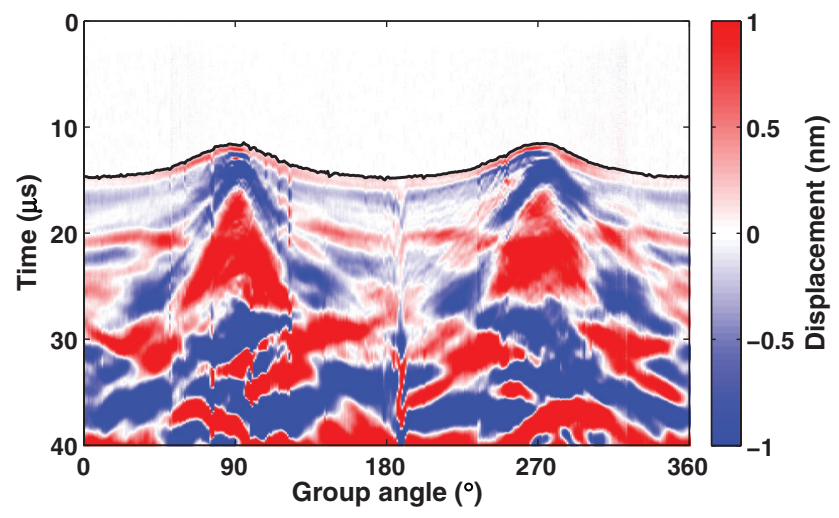

Figure 4. Laser ultrasonic waveforms for the MSH sample after normalization and band-pass filtering between $50 \mathrm{kHz}$ and $5 \mathrm{MHz}$. The black line marks the first-break picks and shows the change in P-wave velocity from the slow direction (group angle of $0^{\circ}$ ) to the fast direction (group angle of $90^{\circ}$ ). It is already apparent in this figure that the central frequency of the first arrival increases from the slow to the fast direction (group angle varying from $0^{\circ}$ to $90^{\circ}$ ), and amplitude strength simultaneously increases. This indicates that wave attenuation decreases for this angle dependence. 
slow direction. The observed events correspond to the direct P-wave followed by surface waves and scattered $\mathrm{P}$ - and $\mathrm{S}$-waves arriving at later times. It is clear from Figures 4 and 5 that both samples have significant $\mathrm{P}$-wave velocity anisotropy, and based on the acquisition geometry, there is data symmetry every $180^{\circ}$. The data also show a significant decrease in amplitude in the direction perpendicular to the layering compared to the direction parallel to the layering. Next, we analyze these traveltime and amplitude variations.

\section{Traveltime analysis}

For both samples, we measure the in-plane (horizontal) component of the wavefield in the slow direction established from Figures 4 and 5, respectively, with the laser light that is reflected off axis (Blum et al., 2010). The horizontal component of the waveform is used to estimate the shear wave velocity along the axis of symmetry, $V_{\mathrm{SV} 0}$, from which we estimate $c_{55}$ with equation 1 . The

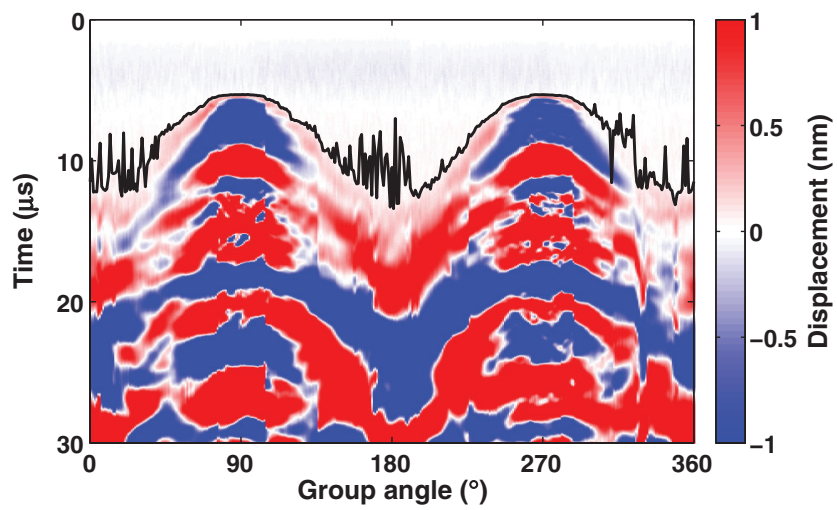

Figure 5. Laser ultrasonic waveforms for the SHC sample after normalization and band-pass filtering between $50 \mathrm{kHz}$ and $5 \mathrm{MHz}$. The black line defines the first-break picks. The attenuation is high along $x_{3}$ (corresponding to a zero group angle) creating difficulties on the automatic picking of first breaks. For this sample, variations in amplitude and frequency content as a function of angle are greater than for sample MSH.

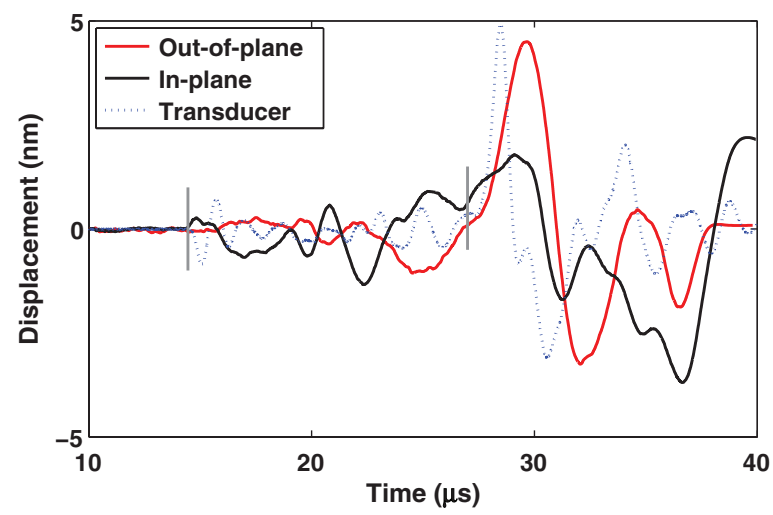

Figure 6. Laser ultrasonic wavefield along the vertical (black) and horizontal (red) components, measured in the slow direction $\left(\theta=\psi=0^{\circ}\right)$. The gray vertical bars mark the first-break pick for each component; we estimate $c_{55}$ from the shear first-break pick. The dotted blue line is a signal acquired using source and receiver shear transducers for comparison. measurement of the in-plane component is very sensitive to the focal position of the laser receiver (Blum et al., 2010). For this reason, we perform a careful measurement of both components in the slow direction $\left(\theta=0^{\circ}\right)$. The measured waveforms on sample MSH are shown in Figure 6 and are compared to source-receiver shear transducer data.

After the relatively straightforward estimation of $c_{11}, c_{33}$, and $c_{55}$ from equations 1 and 2, we invert for $c_{13}$ by performing a leastsquares fit to the measured group velocity data with the theoretical group velocity for a VTI medium using equations 8 and 9. To obtain realistic values for $c_{13}$, we bound our inversion to the theoretical maximum value for $c_{13}$ obtained with equation 7 . This procedure is successfully applied to sample MSH, giving the parameters shown in Table 1 . However, the strong attenuation at $\psi=0^{\circ}$ is preventing us from estimating $c_{55}$ for sample SHC. Because we were unable to estimate $c_{55}$ with laser data on this sample, we perform a joined least-squares fit to estimate $c_{13}$ and $c_{55}$. Figures 7 and 8 show the measured (solid black) and best fit (dashed red) group velocities as a function of group angle for samples MSH and SHC, respectively. Based on these data fits, estimates of the elastic constants for samples MSH and SHC are summarized in Table 1.

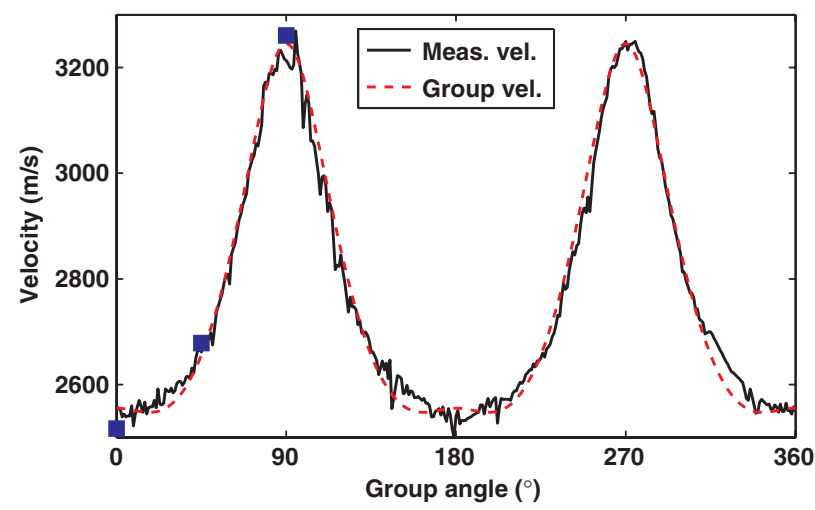

Figure 7. Measured laser ultrasonic P-wave velocity for MSH shale (black solid line). The red dashed line represents the best group velocity fit to the data for $c_{13}=4.1 \pm 1.9 \mathrm{GPa}$. The squares are velocity estimates acquired with transducers at three angles.

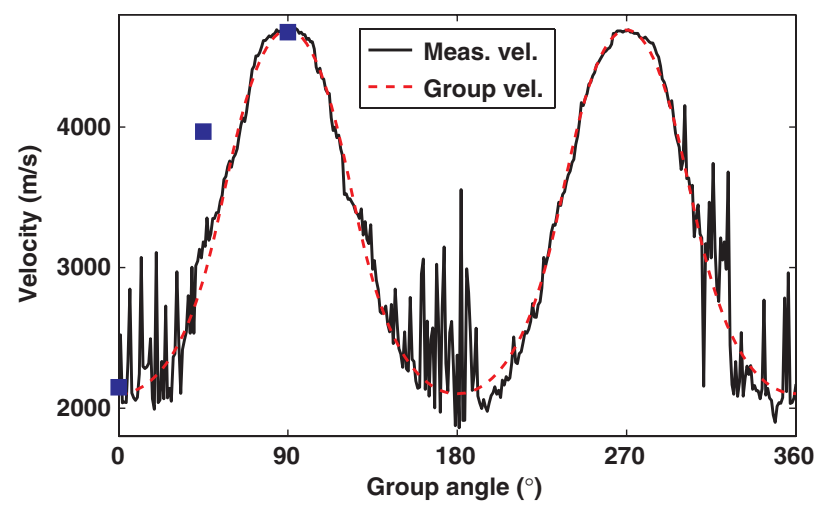

Figure 8. Measured laser ultrasonic P-wave velocity for SHC shale (black solid line). The red dashed line represents the best group velocity fit to the data for $c_{13}=9.5 \pm 0.6 \mathrm{GPa}$ and $c_{55}=11.7 \pm 0.5 \mathrm{GPa}$. Squares are velocity estimates acquired with transducers at three angles. 
We estimate the uncertainties of the measured physical quantities, and we propagate them to the elastic constants. The uncertainties for the constants estimated by a least-squares fit ( $c_{13}$ for both samples and $c_{55}$ for SHC) correspond to the $95 \%$ confidence interval. Although the density seems to play a role in the least-squares fit of equation 5 , it is not the case for velocity data, because measured velocities are converted to $c_{i j}$ 's and then back into velocity, resulting in density cancellation.

We also use transducers to measure the compressional wavefield at $0^{\circ}, 45^{\circ}$, and $90^{\circ}$. The resulting velocities are marked with squares in Figures 7 and 8. For sample SHC, the transducer velocity for $\psi=45^{\circ}$ is $34 \%$ higher than the measured laser ultrasonic velocity.

\section{Amplitude analysis}

We also study the wave attenuation anisotropy for sample MSH. Figure 9 is the extracted absolute amplitude of the trough following the first break after band-pass filtering the processed laser ultrasonic data between 150 and $250 \mathrm{kHz}$. To get a qualitative analysis of the attenuation anisotropy, we compare the experimental amplitudes to the theoretical expression derived in the weak-attenuation anisotropy approximation from equation 36 in Zhu and Tsvankin (2006)

$$
\mathcal{A}_{P}=\mathcal{A}_{P 0}\left(1+\delta_{Q} \sin ^{2} \theta \cos ^{2} \theta+\epsilon_{Q} \sin ^{4} \theta\right),
$$

where $\mathcal{A}_{P 0}$ is the normalized attenuation coefficient giving the decay per wavelength in the symmetry direction $\left(x_{3}\right) . \delta_{Q}$ and $\epsilon_{Q}$ are unitless Thomsen-style parameters defined in Zhu and Tsvankin (2006), in which "the parameter $\delta_{Q}$ is responsible for the attenuation coefficient in near-vertical directions, whereas $\epsilon_{Q}$ controls $\mathcal{A}_{P}$ near the horizontal plane." The attenuation coefficient is often estimated using the spectral-ratio method, requiring an additional amplitude measurement under identical conditions for a nonattenuative reference sample (Zhu et al., 2007). However, our laser source characteristics depend on the sample properties, and they would be different on a reference sample. This prevents us from estimating $\mathcal{A}_{P 0}$ and thus from getting absolute attenuation. Instead, we fit the relative attenuation coefficient to estimate the Thomsenstyle attenuation parameters. We use the symmetry of the setup to average the two halves of the amplitude data, and then we apply a smoothing running average normalized by the group wavenumber. Finally, a least-squares inversion gives the best-fitting parameters with a $95 \%$ confidence interval as $\delta_{Q}=-0.80 \pm 0.23$ and $\epsilon_{Q}=-0.67 \pm 0.03$, and the resulting fit is shown in Figure 10 .

Table 1. Summary of the elastic constants (in GPa) and corresponding anisotropy Thomsen parameters (unitless) for each sample.

\begin{tabular}{lcccc}
\hline Sample & $c_{11}$ & $c_{33}$ & $c_{13, \text { est }}$ & $c_{13, \text { max }}$ \\
\hline MSH & $18.0 \pm 0.4$ & $11.1 \pm 0.2$ & $4.1 \pm 1.9$ & 14.2 \\
SHC & $52.8 \pm 2.6$ & $8.8 \pm 0.3$ & $9.5 \pm 0.6$ & 21.5 \\
Sample & $c_{55}$ & $\epsilon$ & $\delta$ & \\
MSH & $3.3 \pm 0.1$ & $0.31 \pm 0.02$ & $-0.27 \pm 0.22$ & \\
SHC & $11.7 \pm 0.5$ & $2.52 \pm 0.19$ & $6.62 \pm 0.43$ & \\
\hline
\end{tabular}

\section{DISCUSSION}

Laser-based ultrasonic measurements of shale (or other VTI media) anisotropy offer several advantages over a traditional setup with contacting transducers. The technique presented requires only one core drilled perpendicular to the axis of symmetry of the shale. Moreover, the measurements are noncontacting with a small receiver footprint. This allows us to record densely spaced waveforms under computer control. The resulting waveforms provide estimates of the group velocity. Dellinger and Vernik (1994) discuss whether transducer transmission experiments are more likely to measure group or phase velocity. They conclude - based on geometrical arguments - that velocity measurements on the core should yield the phase velocity when the ratio of travel distance $H$ to transducer width $D$ is $H / D<3$, whereas the measurements yield the group velocity when $H / D>20$. Unlike contacting transducers, our ultrasonic laser receiver has a small footprint on the order or $50 \mu \mathrm{m}$, resulting in $H / D \gg 100$ for both samples, clearly yielding the group velocity from this criterion. Note that with the small laser receiver spot size, we avoid the "gray zone" $3<H / D<20$ encountered by many transducer experiments on the core. This zone poses

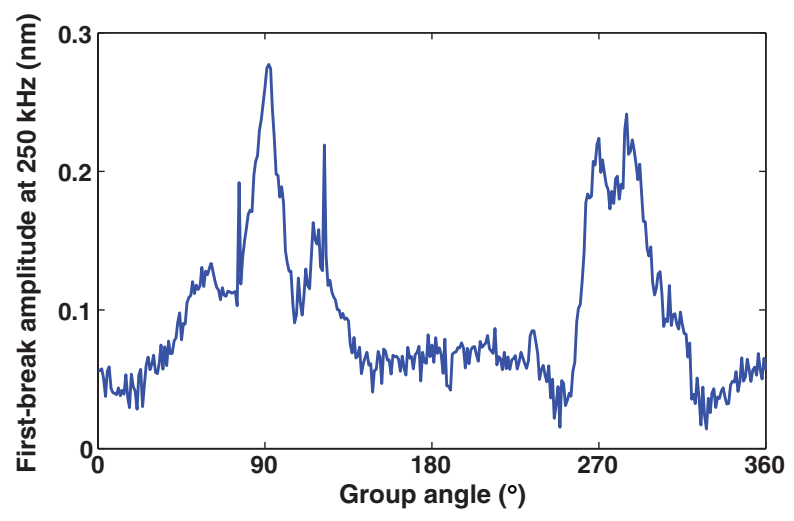

Figure 9. Amplitude of the first break for sample MSH. The lack of smoothness of the curve is due to the uncertainties in the amplitude measurement. However, we can distinctly identify two highamplitude peaks for group angles $90^{\circ}$ and $270^{\circ}$, corresponding to the $x_{1}$-direction and consistent with velocity anisotropy observations.

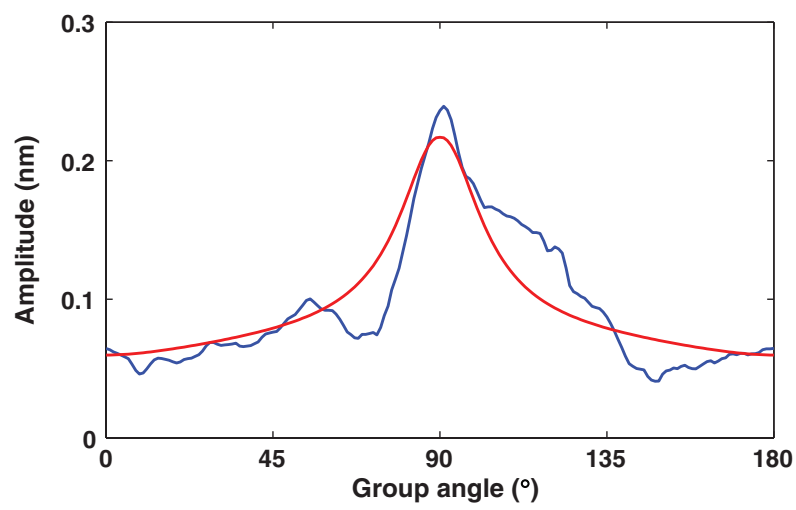

Figure 10. Experimental amplitude smoothed with a running average (blue) and wave attenuation anisotropy least-squares fit (red) for sample MSH, using equation 10 . The best fit is obtained for $\delta_{Q}=$ $-0.80 \pm 0.23$ and $\epsilon_{Q}=-0.67 \pm 0.03$. 
difficulty in the interpretation of our transducer measurements. The transducer setup for sample SHC has $H / D \approx 4$, close to the transition from group to phase velocity. As a result, the velocity estimated from the transducer at $\theta=45^{\circ}$ is close to the expected phase velocity, but it deviates from our group velocity estimation with the laser method. For sample MSH, the ratio $H / D \approx 6$ is also in the transition zone, but we do not observe significant velocity difference at $\theta=45^{\circ}$ when comparing laser and ultrasonic data. This may be because the anisotropy of this sample is weaker, so that the phase and group velocity differ less than for sample SHC.

We checked if our samples are truly VTI materials with the symmetry axis corresponding to our laboratory acquisition coordinate system. We measure the $\mathrm{P}$-wave velocity propagation on the $x_{2}$-axis by placing the laser source and receiver on the sides of the samples parallel to the $\left(x_{1}, x_{3}\right)$ plane (see Figure 2). If the sample is described by a VTI media in the acquisition coordinate system, the $x_{2}$-velocity should be equal in magnitude to the fast $\mathrm{P}$-wave velocity observed in Figures 7 and 8. For both samples, the measurements match closely, confirming that in this case, a VTI representation is an accurate approximation of shale anisotropy.

The observed velocity and amplitude anisotropy in shales can be due to clay mineral composition and alignment (Jones and Wang, 1981; Johnston and Christensen, 1995; Wang, 2002; Dewhurst and Siggins, 2006), layered or lenticular distribution of organic matter and kerogen (Vernik and Nur, 1992; Mba and Prasad, 2010; Sondergeld and Rai, 2011). The negative value of the $\delta$ parameter can be theoretically explained by the alignment and distribution of clay platelets and compliance of the regions between them (Sayers, 2004). The magnitude of our velocity and attenuation anisotropy is almost surely enhanced by delamination and the formation of microfractures parallel to the bedding, resulting from samples drying and in situ stress being released at the surface (Sondergeld and Rai, 2011). In particular, sample SHC has a visible crack parallel to the bedding. As such, the measurements in this publication are meant to illustrate the data acquisition method, and not to be taken as a proper in situ analysis of these particular shale samples.

P-wave amplitude anisotropy is more difficult to measure than velocity anisotropy, but it is shown here to be stronger than velocity anisotropy. In it lies a growing realization that amplitude information has strong potential in understanding the subsurface (Adam et al., 2009), even though reliable amplitude information is typically harder to obtain.

The attenuation of waves in the direction perpendicular to layering observed by Deng et al. (2009) and modeled by Carcione (2000) is in agreement with our observations. Moreover, Zhu et al. (2007) also report large negative values for $\delta_{Q}$ and $\epsilon_{Q}$ in a laboratory study of an anisotropic phenolic sample. We note here that we do not truly satisfy the weak anisotropy conditions assumed in equation 10 because none of $\delta, \epsilon, \delta_{Q}$, or $\epsilon_{Q}$ is $\ll 1$ in modulus. They are, however, small enough to give us a qualitative idea of the attenuation anisotropy.

The data summarized in Table 1 agree with published data at room conditions and on dry core (Vernik and Nur, 1992; Bayuk et al., 2009). With these current results in hand, we aim to expand the methodology to preserved shale cores with minimal alteration to the preserving jacket (wax), while keeping the shale from drying. After that, we will address the problem of making these measurements under in situ reservoir conditions.

\section{CONCLUSIONS}

Noncontacting laser ultrasonics allows us to obtain computercontrolled measurements of the wavefields in shales. These measurements are densely sampled in space and time. This technique reduces problems with cutting samples at angles with a priori unknown symmetry axes, and it provides robust estimates of the fast and slow direction of group velocity. In addition to rock properties obtained from traveltimes, measured amplitudes are absolute and provide attenuation estimates that also relate to the internal structure of the rock. Here, we report anisotropy estimates in velocity and relative attenuation in dry shales, but this is merely a starting point to measurements on preserved samples, eventually under reservoir conditions.

\section{ACKNOWLEDGMENTS}

We thank W. Snyder from Boise State University and C. Sondergeld from the University of Oklahoma for graciously providing samples MSH and SHC, respectively. We also thank D. Dewhurst for his constructive comments, which helped improve this manuscript.

\section{REFERENCES}

Adam, L., M. Batzle, K. T. Lewallen, and K. van Wijk, 2009, Seismic wave attenuation in carbonates: Journal of Geophysical Research, 114, B06208, doi: 10.1029/2008JB005890.

Bayuk, I. O., E. Chesnokov, M. Ammerman, and N. Dyaur, 2009, Elastic properties of four shales reconstructed from laboratory measurements at unloaded conditions: 79th Annual International Meeting, SEG, Expanded Abstracts, 241-245.

Blum, T. E., 2013, Characterization of heterogeneous media with multicomponent laser ultrasonics: Ph.D. thesis, Boise State University.

Blum, T. E., K. van Wijk, B. Pouet, and A. Wartelle, 2010, Multicomponent wavefield characterization with a novel scanning laser interferometer: Review of Scientific Instruments, 81, 073101, doi: 10.1063/1.3455213.

Bretaudeau, F., D. Leparoux, O. Durand, and O. Abraham, 2011, Smallscale modeling of onshore seismic experiment: A tool to validate numerical modeling and seismic imaging methods: Geophysics, 76, no. 5, T101-T112, doi: 10.1190/geo2010-0339.1.

Carcione, J. M., 2000, A model for seismic velocity and attenuation in petroleum source rocks: Geophysics, 65, 1080-1092, doi: 10.1190/1 .1444801 .

Dellinger, J., and L. Vernik, 1994, Do traveltimes in pulse-transmission experiments yield anisotropic group or phase velocities? Geophysics, 59 1774-1779, doi: 10.1190/1.1443564.

Deng, J., S. Wang, and D. hua Han, 2009, The velocity and attenuation anisotropy of shale at ultrasonic frequency: Journal of Geophysics and Engineering, 6, 269-278, doi: 10.1088/1742-2132/6/3/006.

Dewangan, P., I. Tsvankin, M. Batzle, K. van Wijk, and M. Haney, 2006, PSwave moveout inversion for tilted TI media: A physical-modeling study: Geophysics, 71, no. 4, D135-D143, doi: 10.1190/1.2212274.

Dewhurst, D. N., and A. F. Siggins, 2006, Impact of fabric, microcracks and stress field on shale anisotropy: Geophysical Journal International, 165, 135-148, doi: 10.1111/j.1365-246X.2006.02834.x.

Duranti, L., R. Ewy, and R. Hofmann, 2005, Dispersive and attenuative nature of shales: Multiscale and multifrequency observations: 75th Annual International Meeting, SEG, Expanded Abstracts, 1577-1580.

Guilbaud, S., and B. Audoin, 1999, Measurement of the stiffness coefficients of a viscoelastic composite material with laser-generated and detected ultrasound: Journal of the Acoustical Society of America, 105, 2226-2235, doi: 10.1121/1.426827.

Holt, R. M., M. H. Bhuiyan, M. I. Kolstø, A. Bakk, J. F. Stenebraten, and E. Fjær, 2011, Stress-induced versus lithological anisotropy in compacted claystones and soft shales: The Leading Edge, 30, 312-317, doi: 10 $.1190 / 1.3567262$.

Hornby, B. E., 1998, Experimental laboratory determination of the dynamic elastic properties of wet, drained shales: Journal of Geophysical Research, 103, 945-964, doi: 10.1029/97JB02380.

Johnston, J. E., and N. I. Christensen, 1995, Seismic anisotropy of shales: Journal of Geophysical Research, 100, 5991-6003, doi: 10.1029/ 95JB00031. 
Jones, L. E. A., and H. F. Wang, 1981, Ultrasonic velocities in Cretaceous shales from the Williston basin: Geophysics, 46, 288-297, doi: 10.1190/1 .1441199 .

Lebedev, M., A. Bona, R. Pevzner, and B. Gurevich, 2011, Elastic anisotropy estimation from laboratory measurements of velocity and polarization of quasi-P-waves using laser interferometry: Geophysics, 76, no. 3, WA83-WA89, doi: 10.1190/1.3569110.

Mba, K., and M. Prasad, 2010, Mineralogy and its contribution to anisotropy and kerogen stiffness variations with maturity in the Bakken shales: 80th Annual International Meeting, SEG, Expanded Abstracts, 2612-2616.

Miller, D. E., S. A. Horne, and J. Walsh, 2012, Precise inversion of logged slownesses for elastic parameters in a gas shale formation: Geophysics, 77, no. 4, B197-B206, doi: 10.1190/geo2011-0334.1.

Ogi, H., N. Nakamura, K. Sato, M. Hirao, and S. Uda, 2003, Elastic, anelastic, and piezoelectric coefficients of langasite: Resonance ultrasound spectroscopy with laser-Doppler interferometry: IEEE Transactions on Ultrasonics, Ferroelectrics and Frequency Control, 50, 553-560, doi: 10.1109/TUFFC.2003.1201468

Pouet, B., and P. N. J. Rasolofosaon, 1990, Seismic physical modeling using laser ultrasonics: 60th Annual International Meeting, SEG, Expanded Abstracts, 841-844.

Sarker, R., and M. Batzle, 2010, Anisotropic elastic moduli of the Mancos B shale - An experimental study: 80th Annual International Meeting, SEG, Expanded Abstracts, 2600-2605.

Sayers, C. M., 2004, Seismic anisotropy of shales: What determines the sign of Thomsen's delta parameter? 74th Annual International Meeting, SEG, Expanded Abstracts, 103-106.
Scales, J. A., and A. E. Malcolm, 2003, Laser characterization of ultrasonic wave propagation in random media: Physical Review E, 67, 046618, doi: 10.1103/PhysRevE.67.046618.

Scruby, C. B., and L. E. Drain, 1990, Laser ultrasonics techniques and applications, 1st ed.: Taylor \& Francis.

Sondergeld, C. H., and C. S. Rai, 2011, Elastic anisotropy of shales: The Leading Edge, 30, 324-331, doi: 10.1190/1.3567264.

Suarez-Rivera, R., S. Green, J. McLennan, and M. Bai, 2006, Effect of layered heterogeneity on fracture initiation in tight gas shales: Presented at the SPE Annual Technical Conference and Exhibition Proceedings, 103327-MS.

Thomsen, L., 1986, Weak elastic anisotropy: Geophysics, 51, 1954-1966, doi: $10.1190 / 1.1442051$

Tsvankin, I., 2001, Seismic signatures and analysis of reflection data in anisotropic media, 29: Pergamon.

Vernik, L., and A. Nur, 1992, Ultrasonic velocity and anisotropy of hydrocarbon source rocks: Geophysics, 57, 727-735, doi: 10.1190/1.1443286.

Wang, Z., 2002, Seismic anisotropy in sedimentary rocks, part 1: A singleplug laboratory method: Geophysics, 67, 1415-1422, doi: 10.1190/1 .1512787 .

Zhu, Y., and I. Tsvankin, 2006, Plane-wave propagation in attenuative transversely isotropic media: Geophysics, 71, no. 2, T17-T30, doi: 10.1190/1 .2187792 .

Zhu, Y., I. Tsvankin, P. Dewangan, and K. van Wijk, 2007, Physical modeling and analysis of P-wave attenuation anisotropy in transversely isotropic media: Geophysics, 72, no. 1, D1-D7, doi: 10.1190/1.2374797. 